USTC-ICTS-10-21

\title{
Massive charged particle's tunneling from spherical charged black hole
}

\author{
Yan-Gang Miao ${ }^{1,2, *}$, Zhao Xue ${ }^{1, \dagger}$ and Shao-Jun Zhang ${ }^{1, \ddagger}$ \\ ${ }^{1}$ School of Physics, Nankai University, Tianjin 300071, \\ People's Republic of China \\ ${ }^{2}$ Interdisciplinary Center for Theoretical Study, \\ University of Science and Technology of China, Hefei, Anhui 230026, \\ People's Republic of China
}

\begin{abstract}
We generalize the Parikh-Wilczek scheme to the tunneling of a massive charged particle from a general spherical charged black hole. We obtain that the tunneling probability depends on the energy, the mass and the charge of the particle. In particular, the modified Hawking temperature is related to the charge. Only at the leading order approximation can the standard Hawking temperature be reproduced. We take the Reissner-Nordström black hole as an example to clarify our points of view, and find that the accumulation of Hawking radiation makes it approach an extreme black hole.
\end{abstract}

PACS Number(s): 04.70.Dy, 03.65.Sq, 04.62.+v

Keywords: Black hole, quantum tunneling, Hawking temperature

\footnotetext{
*E-mail: miaoyg@nankai.edu.cn

${ }^{\dagger}$ E-mail: illidanpimbury@mail.nankai.edu.cn

${ }^{\ddagger}$ E-mail: sjzhang@mail.nankai.edu.cn
} 


\section{Introduction}

Whether a black hole can radiate puzzled physicists for a long time after the concept of black holes was put forward. In 1974, Hawking proved [1] that black holes are not really black, and that they radiate energy continuously and have an emission spectrum of a black body. Nowadays, the radiation of black holes is called "Hawking radiation". Although Hawking proposed that the radiation of black holes can be viewed as tunneling, he calculated the emission spectrum in light of quantum field theory in curved spacetime without following the tunneling picture.

In 1999, Parikh and Wilczek presented [2] a concise and direct derivation of the Hawking radiation as a tunneling process. They suggested that the barrier is created by the tunneling particle itself, and utilized the Wentzel-Kramers-Brillouin (WKB) method to compute the tunneling rate semiclassically. That is, the WKB expansion was made to the first order, and the Hawking temperature was then obtained in terms of equating the tunneling rate to the Boltzmann factor. The key point of their scheme is to find out a coordinate system in which the metric is well-behaved for calculations at the event horizon. Following the scheme, they derived the emission spectrum of massless and uncharged particles from spherically symmetric black holes, such as the Schwarzschild black hole and Reissner-Nordström black hole. Their results show that although the radiation is not precisely thermal, the leading order is consistent with the standard Hawking radiation, i.e. the black body spectrum. Therefore, Parikh and Wilczek provided a reasonable scheme for the study of the Hawking radiation as a tunneling process. However, they just considered the massless and uncharged particle's tunneling. Later, an attempt was made [3] for the tunneling of a massive and charged particle, in which the main idea is to introduce the concepts of phase velocity and group velocity and to equate the radial velocity of the tunneling particle to the phase velocity.

In addition, based on the Hamilton-Jacobi equation Srinivasan et al. suggested [4] an alternative approach to study the Hawking radiation as a tunneling process. As this approach is not involved in the trajectory problem, it can be applied to the tunneling particles with mass or charge in a natural way. The approach was later developed to extremal and rotating black holes [5], to Taub-NUT black holes [6] and to quantum tunneling beyond semiclassical approximation [7].

Our motivation is straightforward in this note, i.e. to generalize the Parikh-Wilczek scheme [2] to the tunneling process of massive and charged particles from general spherical charged black holes. Different from the ways given by ref. [4] and ref. [3], we neither circumvent the trajectory problem nor adopt the concepts of phase velocity and group velocity. What we want to do is to follow exactly the idea of Parikh and Wilczek and to derive strictly the equation of radial motion for the massive charged tunneling particle. We note that in 
this situation the trajectory of the particle is time-like due to its mass, and is not geodesic anymore due to the electromagnetic interaction between the particle and black hole. In particular, our result, different from that of ref. [3], can on the one hand recover that of Parikh and Wilczek in the limit of zero charge, and on the other hand ensure the dispersion relation of the tunneling particle, which coincides with the basic requirement that the observer at infinity can detect the particle only on shell.

This note is organized as follows. In section 2, we derive the equation of radial motion for the tunneling particle with mass and charge. In section 3, we calculate the imaginary part of the action of the tunneling particle and then give a general expression of the modified Hawking temperature. In section 4 we take the Reissner-Nordström black hole as an example for the application of our generalization of the Parikh-Wilczek scheme. We obtain the corresponding tunneling rate and the Hawking temperature, which can clarify explicitly our points of view. Finally, we make a conclusion in section 5 .

\section{Radial motion of massive charged particle}

A general static spherical charged black hole with mass $M$ and charge $Q$ is depicted by the following metric and electromagnetic potential,

$$
\begin{aligned}
d s^{2} & =-f(r) d t^{2}+g^{-1}(r) d r^{2}+r^{2} d \Omega^{2} \\
A_{\mu} & =h(r) \delta_{\mu}^{0}
\end{aligned}
$$

where $f(r), g(r)$ and $h(r)$ are functions of the parameters: $M, Q$ and $r$. As was done in ref. [2], it is necessary to work in the Painlevé coordinate in order to calculate the Hawking temperature via particle's tunneling. The Painlevé coordinate can be acquired by the transformation [8],

$$
d t_{p}=d t+\sqrt{\frac{1-g(r)}{f(r) g(r)}} d r
$$

where $t_{p}$ denotes the Painlevé time. The resulting Painlevé line element and electromagnetic potential take the forms,

$$
\begin{aligned}
d s^{2} & =-f(r) d t_{p}^{2}+d r^{2}+2 f(r) \sqrt{\frac{1-g(r)}{f(r) g(r)}} d t_{p} d r+r^{2} d \Omega^{2} \\
A_{\mu} & =h(r) \delta_{\mu}^{0}-h(r) \sqrt{\frac{1-g(r)}{f(r) g(r)}} \delta_{\mu}^{1} .
\end{aligned}
$$

The motion of a particle with mass $m$ and charge $q$ in such a spacetime can be described 
by the following Lagrangian ${ }^{1}$,

$$
\mathcal{L}=\frac{m}{2} g_{\mu \nu} \frac{d x^{\mu}}{d \tau} \frac{d x^{\nu}}{d \tau}+q A_{\mu} \frac{d x^{\mu}}{d \tau},
$$

where $\tau$ is chosen to be the proper time. Substituting the solution eqs. (4) and (5) into the above formula, the Lagrangian can be rewritten as

$$
\mathcal{L}=\frac{m}{2}\left[-f(r) \dot{t}_{p}^{2}+\dot{r}^{2}+2 f(r) \sqrt{\frac{1-g(r)}{f(r) g(r)}} \dot{t} \dot{r}\right]+q h(r) \dot{t_{p}}-q h(r) \sqrt{\frac{1-g(r)}{f(r) g(r)}} \dot{r},
$$

where the dot stands for the derivative with respect to $\tau$, i.e. $\dot{x}^{\mu} \equiv \frac{d x^{\mu}}{d \tau}$. By using the Lagrangian (eq. (7)) and its corresponding Euler-Lagrange equation, we can derive the following equation of motion with respect to coordinate $t_{p}$,

$$
-p_{t_{p}} \equiv-\frac{\partial \mathcal{L}}{\partial \dot{t_{p}}}=m f(r) \dot{t_{p}}-m f(r) \sqrt{\frac{1-g(r)}{f(r) g(r)}} \dot{r}-q h(r) \equiv \omega=\text { const. }
$$

where the minus sign before $p_{t_{p}}$ is present due to the positivity of the energy $\omega$ of the tunneling particle. For a time-like trajectory, we also have

$$
g_{\mu \nu} \dot{x}^{\mu} \dot{x}^{\nu}=-1,
$$

which can be explicitly written as

$$
-f(r){\dot{t_{p}}}^{2}+\dot{r}^{2}+2 f(r) \sqrt{\frac{1-g(r)}{f(r) g(r)}} \dot{t_{r}} \dot{r}=-1
$$

With eqs. (8) and (10), we can obtain

$$
\begin{aligned}
\dot{r} & = \pm \frac{1}{m} \sqrt{\frac{g(r)}{f(r)}(\omega+q h(r))^{2}-m^{2} g(r)}, \\
\dot{t_{p}} & =\frac{1}{m f(r)}\left[(\omega+q h(r)) \pm \sqrt{1-g(r)} \sqrt{(\omega+q h(r))^{2}-m^{2} f(r)}\right],
\end{aligned}
$$

where the upper (lower) sign in eqs. (11) and (12) corresponds to outgoing (ingoing) trajectories. As a consequence, we can determine the radial motion with respect to the Painlevé

\footnotetext{
${ }^{1}$ The Lagrangian can be obtained from the covariant equation of motion,

$$
\frac{d^{2} x^{\mu}}{d \tau^{2}}+\Gamma_{\nu \rho}^{\mu} \frac{d x^{\nu}}{d \tau} \frac{d x^{\rho}}{d \tau}=\frac{q}{m} F_{\nu}^{\mu} \frac{d x^{\nu}}{d \tau} .
$$
}


time $t_{p}$ by using eqs. (11) and (12),

$$
\begin{aligned}
\frac{d r}{d t_{p}} & =\dot{r} \dot{t}^{-1} \\
& = \pm f(r) \frac{\sqrt{\frac{g(r)}{f(r)}(\omega+q h(r))^{2}-m^{2} g(r)}}{(\omega+q h(r)) \pm \sqrt{1-g(r)} \sqrt{(\omega+q h(r))^{2}-m^{2} f(r)}}
\end{aligned}
$$

\section{$3 \quad$ Hawking radiation via particle's tunneling}

To derive the Hawking temperature in the tunneling picture, one has to calculate the imaginary part of the action of the tunneling particle. In ref. [2], this imaginary part for the tunneling particle which crosses the horizon outwards from $r_{\text {in }}$ to $r_{\text {out }}$ is defined as

$$
\begin{aligned}
\operatorname{Im} S & \equiv \operatorname{Im} \int_{r_{\text {in }}}^{r_{\text {out }}} p_{r} d r=\operatorname{Im} \int_{r_{\text {in }}}^{r_{\text {out }}} \int_{0}^{p_{r}} d \tilde{p}_{r} d r=\operatorname{Im} \int_{r_{\text {in }}}^{r_{\text {out }}} \int_{m}^{\omega} \frac{d H}{\frac{d r}{d t_{p}}} d r \\
& =-\operatorname{Im} \int_{r_{\text {in }}}^{r_{\text {out }}} \int_{m}^{\omega} \frac{d \tilde{\omega}}{\frac{d r}{d t_{p}}} d r
\end{aligned}
$$

where the Hamilton equation $\frac{d r}{d t_{p}}=\frac{d H}{d \tilde{p}_{r}}$ has been utilized, and the minus sign appears due to the relation [2]: $H=M-\tilde{\omega}$. Note that the tunneling particle is massive in our case, thus the lower limit of energy integral is no longer zero but the particle's mass. This consideration is indispensable ${ }^{2}$ for a massive tunneling particle and we shall see that it will lead to a nontrivial effect, i.e. a shift with respect to the energy on the emission spectrum (see the last paragraph in section 4 for the details). Moreover, we emphasize that the insertion of the lower limit of mass on the integral in eq. (14) does not affect the contour integration ${ }^{3}$. The reason is obvious because the contour integration is only associated with the integration of $d r$ while the lower limit of mass appears in the integration of $d \tilde{\omega}$. The concrete processing is as follows. We use the Parikh-Wilczek's method [2] which is different from the HamiltonJacobi method adopted in ref. [9], and in light of the former we exchange the order of the integration in eq. (14). When we do the integration of $d r$, the lower energy integral limit

\footnotetext{
${ }^{2}$ If the tunneling particle arrives at infinity and is detected by an observer, the trajectory eq. (11) must be valid there. For an approximately flat metric, we have $f(\infty) \sim g(\infty) \sim 1$, and the electromagnetic potential $A_{\mu}(\infty) \sim 0$ which leads to $h(\infty) \sim 0$ due to the static electromagnetic source, such as the Reissner-Nordström black hole we shall discuss in the next section. As a result, we deduce $\omega>m$ from eq. (11), which coincides with the dispersion relation of the tunneling particle at infinity.

${ }^{3}$ The authors would like to thank the anonymous referee for suggesting a discussion on how the lower limit of mass affects the contour integration here, and suggesting an expression for eq. (32) in power of $q$ and an explanation about the absence of effects on the deformed Hawking temperature caused by the particle's mass in the next section.
} 
existing in the integration of $d \tilde{\omega}$ certainly has no effects. Next, when we do the integration of $d \tilde{\omega}$, the lower energy limit then contributes a constant shift as appeared in eq. (19) up to the first order of $\tilde{\omega}$ (see eq. (18)). As a consequence, the integration in eq. (14) is not sensitive to the limit $m \rightarrow 0$, i.e. one can take this limit before or after integration.

The horizon $r_{h}$ is given by $f\left(r_{h}\right)=g\left(r_{h}\right)=0$ and is a function of $M$ and $Q: r_{h}=$ $r_{h}(M, Q)$. Near the horizon one can expand $f(r)$ and $g(r)$ with respect to the radial coordinate $r$ and to the horizon $r_{h}$ as the center,

$$
\begin{aligned}
f(r) & =f^{\prime}\left(r_{h}\right)\left(r-r_{h}\right)+\mathcal{O}\left(\left(r-r_{h}\right)\right), \\
g(r) & =g^{\prime}\left(r_{h}\right)\left(r-r_{h}\right)+\mathcal{O}\left(\left(r-r_{h}\right)\right) .
\end{aligned}
$$

Substituting the above equation into eq. (13), $\frac{d r}{d t_{p}}$ can be approximately expressed as

$$
\frac{d r}{d t_{p}} \simeq \frac{\sqrt{f^{\prime}\left(r_{h}\right) g^{\prime}\left(r_{h}\right)(\omega+q h(r))^{2}-m^{2}{f^{\prime 2}}^{2}\left(r_{h}\right) g^{\prime}\left(r_{h}\right)\left(r-r_{h}\right)}}{(\omega+q h(r))+\sqrt{1-g^{\prime}\left(r_{h}\right)\left(r-r_{h}\right)} \sqrt{(\omega+q h(r))^{2}-m^{2} f^{\prime}\left(r_{h}\right)\left(r-r_{h}\right)}}\left(r-r_{h}\right) .
$$

Considering the self-gravitation of the tunneling particle, eq. (16) should be modified by the replacement of $M \rightarrow M-\omega$ and $Q \rightarrow Q-q$. Substituting eq. (16) into eq. (14) and switching the order of integration for doing the $r$ integral first, we see that there is a pole at $r=r_{h}$. Completing the $r$ integral by deforming the contour around the pole and using the residue method, we have

$$
\operatorname{Im} S=\pi \int_{m}^{\omega} \frac{2}{\sqrt{f^{\prime}\left(r_{h}\right) g^{\prime}\left(r_{h}\right)}} d \tilde{\omega}
$$

where $r_{h}=r_{h}(M-\tilde{\omega}, Q-q)$ and correspondingly $f^{\prime}\left(r_{h}\right)=f^{\prime}\left(r_{h}(M-\tilde{\omega}, Q-q)\right)$ and $g^{\prime}\left(r_{h}\right)=g^{\prime}\left(r_{h}(M-\tilde{\omega}, Q-q)\right)$. In the following, by taking the tunneling probability as $\Gamma \sim e^{-2 \operatorname{Im} S}$ and equating it to the Boltzmann factor $e^{-\frac{\omega}{T}}$, we can obtain the Hawking temperature from eq. (17).

Now making the expansions,

$$
\begin{aligned}
& f^{\prime}\left(r_{h}(M-\tilde{\omega}, Q-q)\right)=f^{\prime}\left(r_{h}(M, Q-q)\right)-\left.f^{\prime \prime}\left(r_{h}(M, Q-q)\right) \frac{\partial r_{h}}{\partial M}\right|_{\tilde{\omega}=0} \tilde{\omega}+\mathcal{O}(\tilde{\omega}), \\
& g^{\prime}\left(r_{h}(M-\tilde{\omega}, Q-q)\right)=g^{\prime}\left(r_{h}(M, Q-q)\right)-\left.g^{\prime \prime}\left(r_{h}(M, Q-q)\right) \frac{\partial r_{h}}{\partial M}\right|_{\tilde{\omega}=0} \tilde{\omega}+\mathcal{O}(\tilde{\omega}),(18
\end{aligned}
$$

and substituting them into eq. (17), we see that only the leading order terms, $f^{\prime}\left(r_{h}(M, Q-q)\right)$ and $g^{\prime}\left(r_{h}(M, Q-q)\right)$, have contributions to the linear term of $\omega$ in $\operatorname{Im} S$. As a consequence, the Hawking temperature is determined by $f^{\prime}\left(r_{h}(M, Q-q)\right)$ and $g^{\prime}\left(r_{h}(M, Q-q)\right)$ completely. Omitting higher order terms in the expansions, we can finish the integral in eq. (17) straightforwardly,

$$
\operatorname{Im} S=\frac{2 \pi}{\sqrt{f^{\prime}\left(r_{h}(M, Q-q)\right) g^{\prime}\left(r_{h}(M, Q-q)\right)}}(\omega-m) .
$$


Therefore, the tunneling probability takes the form,

$$
\Gamma \sim e^{-2 \operatorname{Im} S}=e^{-\frac{4 \pi}{\sqrt{f^{\prime}\left(r_{h}(M, Q-q) g^{\prime}\left(r_{h}(M, Q-q)\right)\right.}}(\omega-m)} .
$$

By equating $e^{-2 \operatorname{Im} S}$ to the Boltzmann factor $e^{-\frac{\omega}{T}}$, we work out the modified Hawking temperature,

$$
T_{H}=\frac{\sqrt{f^{\prime}\left(r_{h}(M, Q-q)\right) g^{\prime}\left(r_{h}(M, Q-q)\right)}}{4 \pi} .
$$

Note that eq. (21) in the zeroth order of $q$ recovers the standard Hawking temperature for a general static spherical charged black hole. In general, the modified Hawking temperature depends on the tunneling particle's charge.

\section{An example: the Reissner-Nordström black hole}

For the Reissner-Nordström black hole, we have

$$
\begin{aligned}
& f(r)=g(r)=1-\frac{2 M}{r}+\frac{Q^{2}}{r^{2}}=\frac{\left(r-r_{+}\right)\left(r-r_{-}\right)}{r^{2}}, \\
& h(r)=-\frac{Q}{r},
\end{aligned}
$$

where the event horizons $r_{ \pm}$are

$$
r_{ \pm}=M \pm \sqrt{M^{2}-Q^{2}} .
$$

As the tunneling particle crosses the outer horizon, we take $r_{h}=r_{+}$. Therefore, the imaginary part of the action (eq. (17)) takes the form,

$$
\operatorname{Im} S=\pi \int_{m}^{\omega} \frac{2 r_{+}^{2}}{r_{+}-r_{-}} d \tilde{\omega} .
$$

Considering the self-gravitation of the tunneling particle with energy $\tilde{\omega}$ and charge $q$, we obtain the event horizons $r_{ \pm}$, and the initial and final positions of the tunneling particle, $r_{i n}$ and $r_{\text {out }}$, as follows:

$$
\begin{aligned}
r_{ \pm} & =M-\tilde{\omega} \pm \sqrt{(M-\tilde{\omega})^{2}-(Q-q)^{2}}, \\
r_{\text {in }} & =M+\sqrt{M^{2}-Q^{2}}, \\
r_{\text {out }} & =M-\omega+\sqrt{(M-\omega)^{2}-(Q-q)^{2}} .
\end{aligned}
$$


Note that ${ }^{4}$ eqs. (27) and (28) have been used when we derive eq. (25). Substituting eq. (26) into eq. (25) and finishing the integral we have

$$
\begin{aligned}
\operatorname{Im} S= & \pi\left[(M-m)^{2}-(M-\omega)^{2}+(M-m) \sqrt{(M-m)^{2}-(Q-q)^{2}}\right. \\
& \left.-(M-\omega) \sqrt{(M-\omega)^{2}-(Q-q)^{2}}\right] .
\end{aligned}
$$

Eq. (29) implies that the emission spectrum is not precisely thermal. As a result, the tunneling rate has the form,

$$
\Gamma \sim e^{-2 \operatorname{Im} S} \sim e^{\Delta S_{\mathrm{BH}}}
$$

where $\Delta S_{\mathrm{BH}}=S_{\mathrm{BH}}(M-\omega, Q-q)-S_{\mathrm{BH}}(M, Q)$ is the difference of the entropy of the black hole after and before the emission. Expanding $\operatorname{Im} S$ in $\omega, q$ and $m$ to the second order, we obtain,

$$
\begin{aligned}
\operatorname{Im} S= & \frac{\pi\left(M+\sqrt{M^{2}-Q^{2}}\right)^{2}}{\sqrt{M^{2}-Q^{2}}}\left\{\left[1+\frac{q Q\left(3 M^{2}-Q^{2}\right)}{\left(M^{2}-Q^{2}\right)\left(M+\sqrt{M^{2}-Q^{2}}\right)^{2}}\right](\omega-m)\right. \\
& \left.+\frac{\left(M^{2}-Q^{2}\right)^{3 / 2}-M Q^{2}}{\left(M^{2}-Q^{2}\right)\left(M+\sqrt{M^{2}-Q^{2}}\right)^{2}}\left(m^{2}-\omega^{2}\right)+\mathcal{O}\left(\omega^{2}, m^{2}, q^{2}, \omega m, \omega q, m q\right)\right\}
\end{aligned}
$$

From the first order term of the above equation with respect to $\omega$, we work out the corresponding modified Hawking temperature,

$$
\begin{aligned}
T_{H} & =\frac{\sqrt{M^{2}-Q^{2}}}{2 \pi\left[\left(M+\sqrt{M^{2}-Q^{2}}\right)^{2}+q Q \frac{3 M^{2}-Q^{2}}{M^{2}-Q^{2}}\right]} \\
& =\frac{\sqrt{M^{2}-Q^{2}}}{2 \pi\left(M+\sqrt{M^{2}-Q^{2}}\right)^{2}}\left[1-\frac{\left(3 M^{2}-Q^{2}\right) Q}{\left(M^{2}-Q^{2}\right)\left(M+\sqrt{M^{2}-Q^{2}}\right)^{2}} q+\mathcal{O}(q)\right]
\end{aligned}
$$

In the following we analyze the characteristics of our result from both the charge and mass viewpoints.

If we set $q=0$ in the above equation, which corresponds to a neutral tunneling particle, we obtain the standard Hawking temperature. For the Reissner-Nordström black hole we give the concrete relation between the modified Hawking temperature and the charge of the tunneling particle. This relation implies an interesting inequality, $\left.T_{H}\right|_{q Q<0}>\left.T_{H}\right|_{q=0}>\left.T_{H}\right|_{q Q>0}$,

\footnotetext{
${ }^{4}$ It is obvious that we have $r_{\text {in }}>r_{h}>r_{\text {out }}$ from eqs. (26), (27) and (28), which gives rise to a pole at $r=r_{h}$ in the integrand of eq. (14). Therefore, following the procedure for deriving eq. (17) from eq. (14) we obtain eq. (25) for the Reissner-Nordström black hole.
} 
which shows that the Reissner-Nordström black hole is more likely to emit particles with the charge opposite to that of the black hole because a higher temperature corresponds to a larger tunneling rate. The accumulation of radiation increases the charge of the black hole and finally makes it equal the mass of the black hole. Consequently, the fate of the Reissner-Nordström black hole is an extreme black hole rather than a Schwarzschild black hole if we only consider the effect caused by the Hawking radiation. This is the way that the charge of the tunneling particle affects the Reissner-Nordström black hole in accordance with the modified Hawking temperature, which is novel and different from that of ref. [3]. Incidentally, we have made a reasonable assumption $|q / Q| \ll 1$ when we derive the temperature expression eq. (32) from eq. (29), that is, the charge of each tunneling particle should be much smaller than that of the black hole. As a result, the temperature eq. (32) is certainly positive.

In addition, it seems to be natural that the particle mass appears in the tunneling rate $\Gamma \sim e^{-2 \operatorname{Im} S}$. Because the spacetime tends to the Minkowski type at infinity, the observer there detects particles only on shell. As the conserved energy $\omega$ can be identified with the energy of the tunneling particle at infinity, i.e. the dispersion relation is satisfied, $\omega$ is definitely larger than the mass $m$. This ensures the consistency of eq. (30), which means $\operatorname{Im} S>0$. With eq. (31) in its first order approximation, we see that the mass produces a shift with respect to the energy $\omega$ on the emission spectrum. This is also different from that of ref. [3]. We note that it is not an artifact of the expansion that the mass does not correct the deformed Hawking temperature. As we can see from eq. (29) which corresponds to the expression before being expanded, the introduction of the mass in $\operatorname{Im} S$ has no influence on the coefficient of $\omega$. (When $m=0$, eq. (29) reduces to the formulation associated with the tunneling of massless particles.) Precisely speaking, the Hawking temperature is proportional to the inverse of the coefficient of $\omega$ in $\operatorname{Im} S$ (eq. (29)). As a result, the mass of the particle has no effects on the modified Hawking temperature. However, it is not trivial that the mass produces no effects on the modified Hawking temperature but the shift with respect to the energy $\omega$ on the emission spectrum. That is, the non-triviality of the effect of the mass corresponds to the physical explanation that the particle can be detected only on-shell at infinity.

\section{Conclusion}

In this note, we discuss the Hawking radiation of a general spherical charged black hole by following the idea of ref. [2] but generalizing it to a quantum tunneling particle with mass and charge. The mass of the tunneling particle gives rise to a time-like trajectory, and the trajectory is not geodesic because of the electromagnetic interaction between the 
charged tunneling particle and black hole. Furthermore, when we change the momentum integral into the energy integral, see eq. (14), we have to impose a nonzero lower limit of energy integral $m$, see the clarifications under eq. (14). We give the formula of the modified Hawking temperature which depends on the charge of the tunneling particle (see eq. (21)).

Taking the Reissner-Nordström black hole as an example, we compute the corresponding tunneling rate and modified Hawking temperature, and find that our result is different from that of ref. [3]. In our result both the mass and charge of the tunneling particle have effects on the emission spectrum which is not precisely thermal either as was first pointed out in ref. [2]. To the first order approximation, we deduce a charge-modified Hawking temperature (see eq. (32)) which recovers the standard Hawking temperature in the limit of zero charge. The modified temperature indicates that the Reissner-Nordström black hole approaches an extreme black hole under the accumulation of the Hawking radiation effect. As to the effect caused by the mass of the tunneling particles, a shift with respect to the energy is produced on the emission spectrum. As we know, the observer at infinity can detect the tunneling particles only on shell. Consequently, the emission spectrum is expected to have a lower cutoff compared with the massless emission spectrum.

Actually we provide a way to realize the idea of ref. [2] for massive and charged tunneling particles. We are considering to apply our method to other kinds of black holes, and shall give the results separately.

\section{Acknowledgments}

Y-GM would like to thank J.-X. Lu of the Interdisciplinary Center for Theoretical Study (ICTS), University of Science and Technology of China (USTC) for warm hospitality. This work is supported in part by the National Natural Science Foundation of China under grants No.11175090 and No.10675061. 


\section{References}

[1] S.W. Hawking, Black hole explosions, Nature 248 (1974) 30;

S.W. Hawking, Particle creation by black holes, Commun. Math. Phys. 43 (1975) 199 [Erratum-ibid. 46 (1976) 206].

[2] M.K. Parikh and F. Wilczek, Hawking radiation as tunneling, Phys. Rev. Lett. 85 (2000) 5042 [arXiv:hep-th/9907001];

M.K. Parikh, A secret tunnel through the horizon, Int. J. Mod. Phys. D 13 (2004) 2351; Gen. Rel. Grav. 36 (2004) 2419 [arXiv:hep-th/0405160].

[3] J.-Y. Zhang and Z. Zhao, Hawking radiation of charged particles via tunneling from the Reissner-Nordström black hole, JHEP 10 (2005) 055.

[4] K. Srinivasan and T. Padmanabhan, Particle production and complex path analysis, Phys. Rev. D 60 (1999) 024007 [arXiv:gr-qc/9812028].

[5] M. Angheben, M. Nadalini, L. Vanzo and S. Zerbini, Hawking radiation as tunneling for extremal and rotating black holes, JHEP 05 (2005) 014 [arXiv:hep-th/0503081].

[6] R. Kerner and R.B. Mann, Tunneling, temperature, and Taub-NUT black holes, Phys. Rev. D 73 (2006) 104010 [arXiv:gr-qc/0603019].

[7] R. Banerjee and B.R. Majhi, Quantum tunneling beyond semiclassical approximation, JHEP 06 (2008) 095 [arXiv:0805.2220[hep-th]].

[8] P. Painlevé, La mécanique classique er la thorie de relativité, C. R. Acad. Sci. (Paris) 173 (1921) 677.

[9] S. Stotyn, K. Schleich and D. Witt, Observer dependent horizon temperatures: a coordinate-free formulation of Hawking radiation as tunneling, Class. Quant. Grav. 26 (2009) 065010 [arXiv:0809.5093[gr-qc]]. 